$\begin{aligned} & \text { Aksaray University } \\ & \text { Journal of Science and Engineering } \\ & \text { e-ISSN: 2587-1277 }\end{aligned}$
http://dergipark.gov.tr/asujse
http://asujse.aksaray.edu.tr

Research Article

\title{
Ullmann Type N-Arylation Reactions of Iodo Pyrazoles with Phenyl Boronic Acid in $\mathrm{Cu}_{2} \mathrm{O}$ Catalyst
}

Melek Hinis", Ayfer Mentes

Aksaray University, Science and Literature Faculty, Department of Chemistry, Aksaray, 68100, Turkey,

-Received Date: 29 Oct 2018

-Revised Date:26 Dec 2018

-Accepted Date:03 Apr 2019

-Published Online:26 May 2019

\begin{abstract}
Ullmann type reactions are one of the most effective methods to carry out the $\mathrm{N}$-arylation of the pyrazole using copper catalyst. The Ullmann reaction carried out by conventional methods occur under hard reaction conditions using copper reagents at stoichiometric ratio and at high temperatures. With the development of more efficient copper catalysts, studies in this field have been continued and very efficient copper/ligand systems have been developed under mild conditions. Although it is known that many of copper / ligand system reactions are carried out under relatively mild conditions to increase the efficiency of Ullmann type reactions, in recent years, studies have been made on simpler catalyst systems which are ligand free and "green" solvents or solvent free.

In this study, in order to contribute to $\mathrm{N}$-arylation of pyrazoles in mild conditions, 4-Iodo-1phenyl -1H-pyrazole $(\mathrm{Ph}-\mathrm{IPz})$ and 4-iodo-3,5-dimethyl-1-phenyl-1H-pyrazole (Ph-IDMPz) compounds were synthesized according to the methods adapted from literature with $\mathrm{N}$-arylation reaction of 4-Iodo-1 $H$-pyrazole (4-IP) or 4-iodo-3,5-dimethyl- $1 H$-pyrazole (4-IDMPz) with phenyl boronic acid in the prences of $\mathrm{Cu}_{2} \mathrm{O}$ catalyst. $\mathrm{N}$-Arylation reactions of 4-iodopyrazols with phenyl boronic acit were carried out the presence of $\mathrm{Cu}_{2} \mathrm{O}$ catalyst, at atmospheric conditions, at room temperature, in methyl alcohol and without the use of any base and ligand. In this way, the synthesis of Ph-IPz and Ph-IDMPz was carried out for the first time with Ullmann reaction under the milder conditions.

Synthesized ligands Ph-IPz and Ph-IDMPz were characterized by FT-IR, NMR and GC-MS mass spectra. The NMR and FT-IR spectra of the compounds showed characteristic peaks of both the pyrazole and the phenyl group. The absence of N-H proton peaks in the $1 \mathrm{H}-\mathrm{NMR}$ spectra of the obtained Ph-IP 2 and Ph-IDMP 2 compounds indicates that the phenyl group is attached from the pyrazole $\mathrm{NH}$. The shifts in the $\mathrm{C} 4$ carbon peaks which are determinant for 4substituted pyrazoles in the 13C-NMR spectra also confirm the structure of the compounds.
\end{abstract}

\section{Keywords}

Pyrazole, N-arylation, Copper, Boronic acid

*Corresponding Author: Melek Hinis, melekhinis@ gmail.com 


$\begin{aligned} & \text { Aksaray University } \\ & \text { Journal of Science and Engineering } \\ & \text { e-ISSN: 2587-1277 }\end{aligned}$
http://dergipark.gov.tr/asujse
http://asujse.aksaray.edu.tr

Research Article

\section{Iyodo Pirazollerin $\mathrm{Cu}_{2} \mathrm{O}$ Katalizörlüğünde Fenil Boronik Asit ile Ullmann Tipi N-Arilasyon Reaksiyonları}

Melek Hinıs*, Ayfer Mentes

Aksaray Üniversitesi, Fen Edebiyat Fakültesi, Kimya Bölümü, Aksaray, 68100, Türkiye

-Gönderi Tarihi: 29 Eki 2018

-Düzeltme Tarihi:26 Ara 2018

-Kabul Tarihi:03 Nisan 2019

-Çevrimiçi Yayın Tarihi:26 May 2019

\section{Özet}

Ullmann tipi reaksiyonlar bakır katalizörü kullanılarak pirazollerin $\mathrm{N}$-arilasyonu gerçekleştirmek için en etkili yöntemlerden birisidir. Geleneksel yöntemlerle gerçekleştirilen Ullmann reaksiyonu yüksek sıcaklıklarda ve stokiyometrik oranda bakır reaktifleri kullanılarak sert reaksiyon koşulları altında gerçekleşmektedir. Daha verimli bakır katalizörlerinin gelişmesi ile bu alanda yapılan çalışmalar devam ederek hafif koşullar altında oldukça verimli bakır/ligant sistemleri geliştirildi. Ullmann tipi reaksiyonların verimliliğini arttırmak için birçok bakır/ligant kombinasyon reaksiyonlarının nispeten yumuşak koşullar altında gerçekleştirdiği bilinmesine rağmen, son yıllarda ligant içermeyen ve "yeşil" çözücülerle ya da çözücüsünden arındırılmış daha basit katalizör sistemleri üzerine çalışmalar yapılmaktadır.

$\mathrm{Bu}$ çalışmada pirazollerin ılıman şartlarda N-arilasyonuna katkı sağlaması amacıyla fenil boronik asit ile 4-iyodo-1 $H$-pirazol (I-Pz) veya 4-iyodo-3,5-dimetil-1H-pirazolün (I-DMPz) $\mathrm{Cu}_{2} \mathrm{O}$ katalizörlüğünde $\mathrm{N}$-arilasyonu ile 4-Iyodo-1-fenil-1H-pirazol (Ph-IPz) ve 4-Iyodo-3,5dimetil-1-fenil-1H-pirazol (Ph-IDMPz) bileşikleri literatürdeki yönteme göre uyarlanarak sentezlenmiştir. 4-İyodo pirazollerin fenil boronik asit ile $\mathrm{N}$-arilleme reaksiyonları $\mathrm{Cu}_{2} \mathrm{O}$ katalizörlüğünde, atmosferik koşullarda, oda sıcaklığında ve metil alkol içerisinde herhangi bir baz ve ligant kullanılmadan gerçekleştirilmiştir. Böylece daha 1lıman koşullarda Ullmann reaksiyonu ile ilk defa Ph-IPz ve Ph-IDMPz'ın sentezi gerçekleştirilmiştir.

Sentezi gerçekleşen $\mathrm{Ph}-\mathrm{IPz}$ ve Ph-IDMPz'in yapıları FT-IR, NMR ve GC-MS kütle spektrumları alınarak karakterize edilmiştir. Bileşiklerin NMR ve FT-IR spektrumunda hem pirazole hem de fenil grubuna ait karakteristik pikler gözlenmiştir. Elde edilen $\mathrm{Ph}-\mathrm{IPz}$ ve $\mathrm{Ph}-$ IDMPz bileşiklerinin ${ }^{1} \mathrm{H}-\mathrm{NMR}$ spektrumlarında N-H proton piklerinin görülmemesi fenil grubunun pirazole NH'dan bağlandığının bir göstergesidir. ${ }^{13} \mathrm{C}-\mathrm{NMR}$ spektrumlarında 4sübstitüe pirazoller için belirleyici olan $\mathrm{C}_{4}$ karbon piklerindeki kaymalar da bileşiklerin yapılarını doğrulamaktadır.

\section{Anahtar Kelimeler}

Pirazol, N-arilasyon, Bakır, Boronik asit

*Sorumlu Yazar: Melek Hınıs, melekhinis@gmail.com 


\section{GÍRISS}

Pirazol, beş üyeli halka yapısı ile karakterize edilen heterosiklik halkalı organik bileşikler sınıfındandır [1]. Pirazol türevlerinin sergilediği ilginç özelliklerinin keşfedilmesiyle, pirazol kimyasına ilgi önemli ölçüde artmıştır. Pirazol ve pirazol türevleri antibakteriyel, antifungisital, antiherbisital, antiinsektisital ve diğer biyolojik aktivitelerin yanı sıra antitümör, antimikrobiyal anti-enflamatuar, analjezik ajanlar gibi oldukça geniş biyolojik ve farmakolojik etkinliklere sahip olmaları nedeniyle araştırma konusu olmaya devam etmektedir [2, 3].

Son yıllarda pirazol türevi bileşiklerin hazırlanmasında seçiciliği artırmak için genel ve verimli metotlar geliştirilmiştir. Bunların arasında sübstitüye pirazoller ile aril elektrofillerin çapraz bağlanma tepkimeleri pirazol türevlerinin sentezlenmesinde geleneksel metotlara karşı alternatif olarak ortaya çıkmıştır [4].

$\mathrm{N}$-Aril pirazoller, yıllardır 1,3-diketonların aril hidrazinler ile gerçekleştirdiği reaksiyon sonucu sentezlenmektedir [5]. Bu yönteme alternatif olarak $1 \mathrm{H}$-pirazollerin aril halojenür, hetero (Het) aril halojenür veya aril boronik asitlerle elektrofilik olarak geçiş metali katalizli $\mathrm{N}$-arillenmesi yöntem olarak geliştirilmiştir (Şekil 1). Bu yöntem birçok doğal ürünün ve tıbbi maddenin sentezlenebilmesi için gerekli yapı taşları olan ürünlerin sentezlenmesinde büyük bir rol oynamıştır [4].<smiles></smiles>

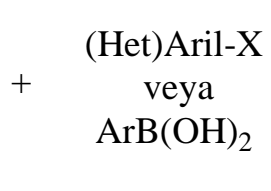
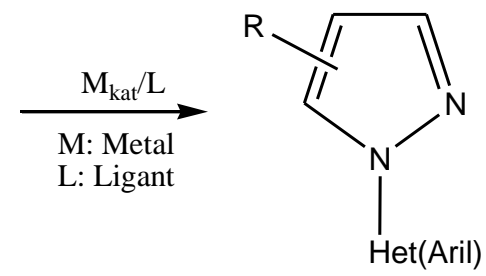

Şekil 1. Pirazolün geçiş-metal katalizli N- arilleme reaksiyonu [8].

Azot içeren heterosiklik yapıların aril halojenürler ile geçiş metal katalizli arilasyonu, Narilazol türevlerinin sentezi için en etkili ve güçlü yöntemlerden biridir ve bu yöntemin hem akademik hem de endüstriyel laboratuvarlarda yararlı olduğu kanıtlanmıştır. Ancak, mevcut yöntemlerde kullanılan paladyum, rodyum, nikel ve kobalt kompleksleri gibi pahalı geçiş metal komplekslerinin bazı sınırlamaları vardır. Arilasyon reaksiyonu için ucuz ve çevre açısından iyi hâle getirilen metal katalizörlerin kullanılması tercih edilmektedir [6].

Pirazollerin veya diğer heterosiklik yapıların bakır katalizli N-arilasyonu (Ullmann tipi reaksiyon), $\mathrm{C}_{(\mathrm{ar})}-\mathrm{N}$ bağları oluşturmak için en etkili yöntemlerden birini temsil eder. Ancak geleneksel bakır katalizörlü Ullmann reaksiyonunun $210{ }^{\circ} \mathrm{C}$ 'a kadar yüksek sıcaklıklarda olması ve stokiyometrik oranda bakır reaktifleri kullanılan sert reaksiyon koşullarında 
olmasından dolayı, daha verimli bakır katalizörleri geliştirilmiştir. Bu bağlamda yapılan çalışmalar, muhtemelen artan çözünürlük ve bakır katalizörlerin stabilitesi nedeniyle organik bir katkı maddesinin varlığında arilleme yapıldığında tepkime hızlarının belirgin bir şekilde arttırıldığını ortaya çıkarmıştır. 2001'de Taillefer ve Buchwald'ın araştırma grupları, sırasıyla, hafif koşullar altında metalin katalitik bir miktarının kullanılmasına izin veren oldukça verimli bakır/ligant sistemlerini keşfettiler [7, 8]. O zamandan beri, birçok araştırma grubu çapraz bağlama reaksiyonlarının verimliliğini arttırmak için yeni bakır/ligant sistemleri geliştirmiştir. Özellikle, bakır katalizörlü sistemlerin düşük maliyeti endüstriyel uygulamalar için ilgi çekici olmaktadır [4].

Genel olarak, bakır katalizli çapraz bağlanma reaksiyonlarında bakırın türünden çok ligant, baz veya çözücünün seçimi daha önemlidir $[9,10]$. $\mathrm{Cu}(\mathrm{I})$ olarak $\mathrm{CuI}$ veya $\mathrm{Cu}_{2} \mathrm{O}$ çoğunlukla kullanılan bakır türleridir. Son yıllarda ek bir ligant olmaksızın $\mathrm{CuO}$ ve hatta $\mathrm{Cu}$ tozu da kullanılmaktadır. Genellikle, baz olarak $\mathrm{Cs}_{2} \mathrm{CO}_{3}$ veya $\mathrm{K}_{2} \mathrm{CO}_{3}$, çözücü olarak DMSO veya DMF içinde reaksiyonlar gerçekleştirilir [4]. Birçok bakır/ligant kombinasyon reaksiyonlarının nispeten yumuşak koşullar altında gerçekleştirdiği bilinmesine rağmen, ligant içermeyen ve "yeşil" çözücülerle ya da çözücüsünden arındırılmış daha basit katalizör sistemleri bulmaya ihtiyaç duyulmaktadır.

$\mathrm{Bu}$ çalışmada literatürdeki yönteme göre sentezlenen [11] 4-iyodo pirazollerin fenil boronik asit ile $\mathrm{Cu}_{2} \mathrm{O}$ katalizörlüğünde $\mathrm{N}$-arilasyonları gerçekleştirildi. $\mathrm{N}$-arilleme reaksiyonu klasik Ullmann reaksiyonunu geliştirerek daha 1lıman koşullarda oluşmasına katkıda sağlamak amacıyla ilave ligant veya baz olmadan, atmosferik koşullarda, oda sıcaklığında metanol içerisinde karıştırılarak literatürdeki yönteme göre uyarlanarak sentezlenmiştir [12-15]. Bu yöntemle pirazol türevleri olan Ph-IDMPz ve Ph-IPz bileşiklerinin sentezi ilk defa bu çalışma ile gerçekleştirilmiş oldu.

\section{MALZEME VE YÖNTEM}

Deneylerde kullanılan tüm kimyasallar Merck ve Sigma Aldrich firmasından temin edilmiş olup herhangi bir saflaştırma yapılmadan olduğu gibi kullanılmıştır. Reaksiyon başlangıç maddeleri I-Pz ve I-DMPz literatürdeki yönteme göre saf pirazol $(\mathrm{Pz})$ veya 3,5-dimetil pirazol (DMPz) kullanılarak sentezlenmiştir [11]. Reaksiyonlarda ve saflaştırma işlemlerinde çözücü olarak metanol veya diklorometan kullanılmıştır. TLC (Gel 60 F254 TLC Alüminyum tabakalı 20x20 cm) kullanılarak reaksiyonlar takip edilmiştir.

Sentezlenen bileşiklerin ${ }^{1} \mathrm{H}$ NMR, ${ }^{13} \mathrm{C}$ NMR spektrumları, Agilent marka $600 \mathrm{MHz}$ 'lik frekansta, 14.1 Tesla alan gücüne sahip Premium Compact NMR cihazı ile yapıldı. FT-IR 
spektrumları Perkin Elmer Spectrum 100 FT-IR spektrometresi ile ATR tekniği kullanılarak 650-4000 $\mathrm{cm}^{-1}$ aralığında kaydedildi. Büchi B-540 marka erime noktası tayin cihazı ile erime noktaları ölçüldü. GC-MS kütle spektrumları Agilent GC-7890 A-MS 5975 marka cihaz ile $30 \mathrm{mx} 250 \mu \mathrm{mx} 0.25 \mu \mathrm{m}$ kolon ile $20 \mathrm{~mL} /$ dak akış hızı kullanılarak alındı.

\subsection{SENTEZLENEN LİGANTLAR}

\subsubsection{4-İyodo-1-fenil-1H-pirazol sentezi, (Ph-IPz)}

Fenil boronik asit ( $1 \mathrm{mmol}, 0.122 \mathrm{~g}), \mathrm{I}-\mathrm{Pz}(1.2 \mathrm{mmol}, 0.232 \mathrm{~g})$ ve $\mathrm{Cu}_{2} \mathrm{O}$ (\%5.5 mmol, pirazol türevine göre) metil alkol ( $5 \mathrm{~mL})$ içerisinde oda sıcaklığında 3 gün karıştırıldı. TLC ile ürün oluşumu kontrol edildi. Karışım süzüldükten sonra çözücüsü buharlaştırıldı. Oluşan madde diklorometanda çözüldü ve su $(2 \times 10 \mathrm{~mL})$ ile yıkandı. Organik kısım ayrıldıktan sonra silikadan süzüldü ve süzüntü $\mathrm{MgSO}_{4}$ ile kurutuldu. Oluşan beyaz kristalin maddenin $\mathrm{Ph}-\mathrm{IPz}$ sentez reaksiyonu Şekil 2'de gösterilmektedir. (E.N: 105-106 ${ }^{\circ} \mathrm{C}$, Verim: \%25).

GC-MS $(m / z): 270$.

FT-IR $\left(A T R, \mathrm{~cm}^{-1}\right):$ 3114-2783 v(C-H)ar, $1598 v(\mathrm{C}=\mathrm{N}), 1534 v(\mathrm{C}=\mathrm{C}), 1364 \mathrm{v}(\mathrm{C}-\mathrm{N}), 1029 v(\mathrm{~N}-$ $\mathrm{N})$.

${ }^{1} \mathrm{H}-\mathrm{NMR}\left(600 \mathrm{MHz}, \mathrm{CDCl}_{3}\right), \delta(\mathrm{ppm}):$ 7.4-7.5 (5H, Ph), $7.9\left(1 \mathrm{H}, \mathrm{C}_{3}-\mathrm{Pz}\right), 8.1\left(1 \mathrm{H}, \mathrm{C}_{5}-\mathrm{Pz}\right)$.

${ }^{13} \mathrm{C}-\mathrm{NMR}$ (150 MHz, $\left.\mathrm{CDCl}_{3}\right), \delta(\mathrm{ppm}): 56.2\left(\mathrm{C}_{4}-\mathrm{Pz}\right), 127-134$ (6C-Ph), $141\left(\mathrm{C}_{3}, \mathrm{C}_{5}-\mathrm{Pz}\right)$.<smiles>Oc1ccccc1</smiles>

Fenil Boronik Asit

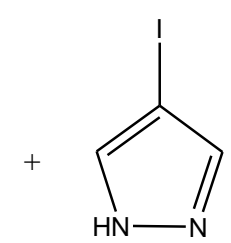

4-iyodo-1H- pirazol

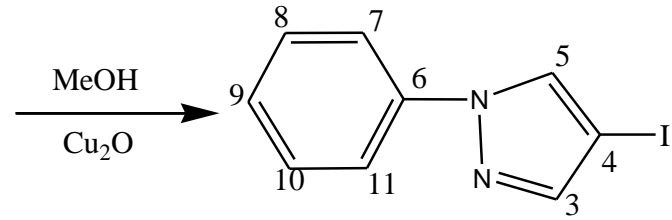

4-iyodo-1-fenil-1 $H$-pirazol

Ph-IPz

Şekil 2. 4-İyodo-1-fenil-1H-pirazolün (Ph-IPz) sentez şeması.

\subsubsection{4-İyodo-3,5-dimetil-1-fenil-1H-pirazol sentezi, (Ph-IDMPz)}

Fenil boronik asit (2.2 mmol, 0.268 g), I-DMPz (2 mmol, $0.488 \mathrm{~g})$ ve $\mathrm{Cu}_{2} \mathrm{O}$ (\%5.5 mmol, pirazol türevine göre) metil alkol (10 mL) içerisinde 35 dak. karıştırıldı. TLC ile ürün kontrol edildi. Karışım süzüldükten sonra çözücüsü buharlaştırıldı ve oluşan madde diklorometanda çözüldü, su $(3 \times 15 \mathrm{~mL})$ ile yıkandı. Tepkime ortamından organik fazı ayrıldıktan sonra silikadan süzüldü ve süzüntü $\mathrm{MgSO}_{4}$ ile kurutuldu. Çözücüsü oda sıcaklığında buharlaştırılan açık kahverengi madde Şekil 3'deki reaksiyona göre elde edildi. (E.N: $180{ }^{\circ} \mathrm{C}$, Verim: \%56).

LC-MS/MS (m/z): 298 [M]. 
FT-IR (ATR, $\left.\mathrm{cm}^{-1}\right):$ 3168-3072 v(C-H)ar, 2921-2836 v(C-H $)_{\mathrm{al}}, 1599 v(\mathrm{C}=\mathrm{N}), \quad 1572-1508$ $v(\mathrm{C}=\mathrm{C}), 1326 v(\mathrm{C}-\mathrm{N}), 1028 v(\mathrm{~N}-\mathrm{N})$.

${ }^{1} \mathrm{H}-\mathrm{NMR}\left(600 \mathrm{MHz}, \mathrm{DMSO}-\mathrm{d}_{6}\right), \delta(\mathrm{ppm}): 2.4\left(6 \mathrm{H}, \mathrm{CH}_{3}\right), 7.2-7.7(5 \mathrm{H}-\mathrm{Ph})$.

${ }^{13} \mathrm{C}-\mathrm{NMR}\left(150 \mathrm{MHz}, \mathrm{DMSO}-\mathrm{d}_{6}\right) \delta(\mathrm{ppm}): 13.6-14.2\left(2 \mathrm{CH}_{3}-\mathrm{Pz}\right), 62.6\left(\mathrm{C}_{4}-\mathrm{I}, \mathrm{Pz}\right), 124-134$ (6C$\mathrm{Ph}), 141\left(\mathrm{C}_{5}-\mathrm{Pz}\right), 150\left(\mathrm{C}_{3}-\mathrm{Pz}\right)$.

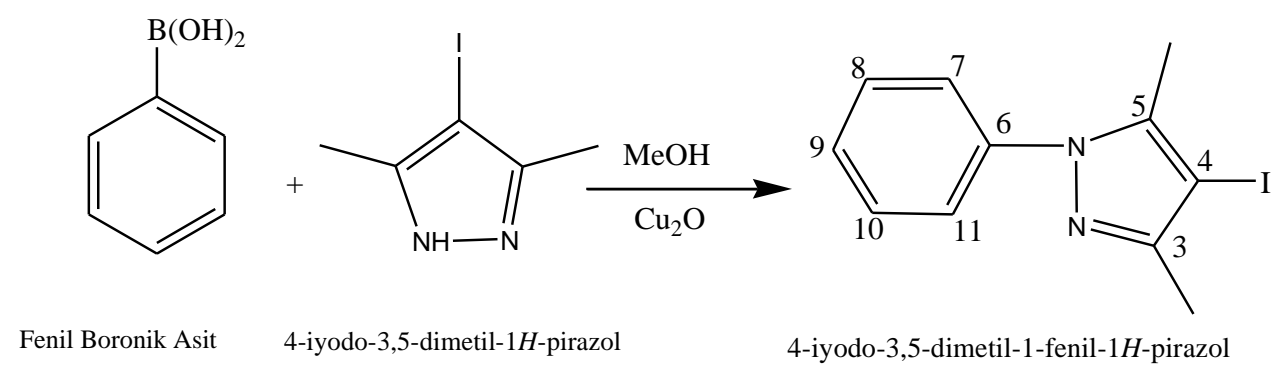

(Ph-IDMPz)

Şekil 3. 4-İyodo-3,5-dimetil-1-fenil-1H-pirazolün (Ph-IDMPz) sentez şemas1

\section{BULGULAR VE TARTIŞMA}

Ullmann yöntemi ile sentezlenen Ph-IPz ve Ph-IDMPz ligantları organik polar çözücülerde çözünmektedir. Bu ligantlar dietil eter, hekzan, petrol eteri gibi apolar organik çözücülerde ve suda çözünmemektedir.

Tablo 1'de sentezlenen ligantların, analitik ve spektroskopik özellikleri, GC-MS, FT-IR ve NMR spektrumları ise Şekil 4- Şekil 11'de verilmiştir. Ph-IPz ve Ph-IDMPz ligantlarının kütle spektrumları ile molekül ağırlıklarının önerilen yapılar ile uyumlu olduğu Tablo 1'de görülmektedir.

Ph-IPz'ün sentez veriminin Ph-IDMPz'göre daha düşük olduğu görülmektedir. Başlangıç maddesi I-DMPz'ün fenil boronik asit ile reaksiyonu, I-Pz'ün reaksiyonu ile kıyaslandığında hem veriminin daha fazla olduğu hem de reaksiyonun daha kısa sürede tamamlandığ görülmüştür. I-DMPz'ün yapısında elektron verici metil gruplarının bağlı olması ve metil gruplarının pirazol halkasında simetrik etkiye sahip olmasından dolayı reaksiyonun daha hızlı ve verimin daha yüksek olmasını sağlamaktadır.

Tablo 1: Sentezlenen ligantların özellikleri

\begin{tabular}{|c|c|c|c|c|c|}
\hline Ligant & Renk & Verim (\%) & E.N. $\left({ }^{\circ} \mathbf{C}\right)$ & MA (g/mol) & Kütle \\
\hline Ph-IPz & Beyaz & 25 & $105-106$ & 270 & $270[\mathrm{M}]$ \\
\hline Ph-IDMPz & Açı Kahve & 56 & 180 & 298 & $298[\mathrm{M}]$ \\
\hline
\end{tabular}

Ph-IPz'ün erime noktası $105-106{ }^{\circ} \mathrm{C}$ iken Ph-IDMPz'nin $180{ }^{\circ} \mathrm{C}$ 'de ölçülmüştür. Başlangıç maddeleri I-Pz' ün erime noktası $100-104{ }^{\circ} \mathrm{C}$; I-DMPz'ün erime noktası $137-140{ }^{\circ} \mathrm{C}$ 'dir. 
Sentezlenen ürünlerin erime noktalarının başlangıç maddelerinden farklı olduklarından dolayı ürünün saf olarak gerçekleştirildiği görülmektedir.

FT-IR spektrumları incelendiğinde (Tablo 2) sentezlenen ligantların pirazol halkasına bağlı aromatik C-H bandları $3168 \mathrm{~cm}^{-1}$ ile $2783 \mathrm{~cm}^{-1}$ aralığında, Ph-IDMPz'deki alifatik C-H bandlar1 ise 2921-2836 $\mathrm{cm}^{-1}$ aralığında gözlenmiştir. Spektrumlarda pirazol halkasına ait karakteristik bandlar 1599-1598 $\mathrm{cm}^{-1}$ aralığında $\mathrm{C}=\mathrm{N}, 1572-1534 \mathrm{~cm}^{-1}$ aralığında $\mathrm{C}=\mathrm{C}, 1326-1364 \mathrm{~cm}^{-1}$ aralığında C-N ve 1029-1028 $\mathrm{cm}^{-1}$ aralığında N-N gerilme bandları görülmüştür [16-18]. Fenil pirazollerde $\mathrm{C}=\mathrm{N}$ halka gerilmelerinin $1758-1626 \mathrm{~cm}^{-1}$, den $1599-1598 \mathrm{~cm}^{-1}$,e ve $\mathrm{C}=\mathrm{C}$ halka

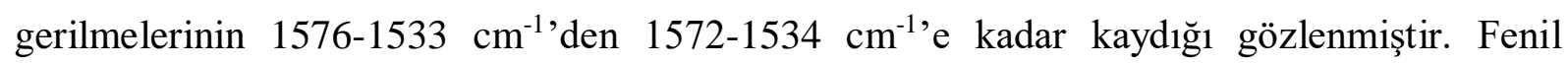
pirazollerde başlangıç maddelerine göre $\mathrm{C}=\mathrm{C}$ ve $\mathrm{C}=\mathrm{N}$ halka gerilmelerindeki kaymalar pirazol halkasına fenil gruplarının bağlanmasının bir göstergesidir.

Tablo 2. Sentezlenen ligantların FT-IR spektrumları $\left(\mathrm{cm}^{-1}\right)$.

\begin{tabular}{|c|c|c|c|c|c|c|c|}
\hline Ligant & U (N-H) & $v(\mathrm{C}-\mathrm{H}) \mathrm{ar}$ & $v(\mathbf{C}-\mathbf{H}) \mathrm{al}$ & $v(\mathbf{C}=\mathbf{N})$ & $v(C=C)$ & $v(\mathbf{C}-\mathrm{N})$ & $v(\mathrm{~N}-\mathrm{N})$ \\
\hline $\mathrm{I}-\mathrm{Pz}$ & 3113 & $3037-2783$ & --- & 1626 & 1533 & 1321 & 1030 \\
\hline I-DMPz & 3164 & --- & $2917-2826$ & 1758 & 1576 & 1305 & 1033 \\
\hline $\mathrm{Ph}-\mathrm{IPz}$ & & $3114-2783$ & --- & 1598 & 1534 & 1364 & 1029 \\
\hline Ph-IDMPz & & $3168-3072$ & $2921-2836$ & 1599 & 1572 & 1326 & 1028 \\
\hline
\end{tabular}

Fenil pirazol ligantlarının NMR spektrumları Şekil 8-11'de verilmiştir. Ph-IPz ligantının ${ }^{1} \mathrm{H}-$ NMR spektrumunda pirazolün $\mathrm{H}_{3}$ ve $\mathrm{H}_{5}$ protonları sirasıyla 8.1 ve $7.9 \mathrm{ppm}$ de, fenil grubuna ait aromatik halka protonları 7.5-7.4 ppm aralığında gözlenmiştir. $\mathrm{Ph}-\mathrm{IPz}$ ligantının ${ }^{13} \mathrm{C}-\mathrm{NMR}$ spektrumunda pirazolün $\mathrm{C}_{3}$ karbonu 141.1 ppm' de, $\mathrm{C}_{5}$ karbonu 133.8 ppm' de, fenil grubuna ait aromatik halka karbonları 134.8-127.4 ppm aralığında gözlemlenmiştir. Pirazolün $\mathrm{C}_{4}$ karbonu literatürdeki değerler ile uyumlu olarak 56.2 ppm' de sinyal vermiştir [6]. Ph-IDMPz ligantının ${ }^{1} \mathrm{H}-\mathrm{NMR}$ spektrumunda fenil grubuna ait aromatik halka protonlar1 7.7-7.3 ppm aralığında, pirazole ait $\mathrm{CH}_{3}$ protonları ise 2.1 ppm'de görülmüştür. $\mathrm{Ph}-\mathrm{IDMPz}$ ligantının ${ }^{13} \mathrm{C}$ NMR spektrumunda pirazolün $\mathrm{C}_{3}$ karbonu 150.1 ppm' de, $\mathrm{C}_{5}$ karbonu ise 141.5 ppm' de sinyaller vermiştir. Fenil grubuna ait aromatik halka karbonları 134.5-124.8 ppm aralığında, pirazolün $\mathrm{C}_{4}$ karbonu da 62.8 ppm' de gözlemlenmiştir [6]. Ph-IDMPz bileşiğindeki 3,5-dimetil pirazole ait $\mathrm{CH}_{3}$ karbonları ise 13.6 ve 14.2 ppm'de görüldüğ ü bu değerler literatürdeki veriler ile uyumlu olduğu tespit edilmiştir [17, 19].

Elde edilen spektroskopik veriler sonucunda $\mathrm{N}$-arilasyon yöntemi ile sentezi gerçekleştirilen $\mathrm{Ph}-\mathrm{IPz}$ ve Ph-IDMPz'nin yapıları doğrulanmıştır. 


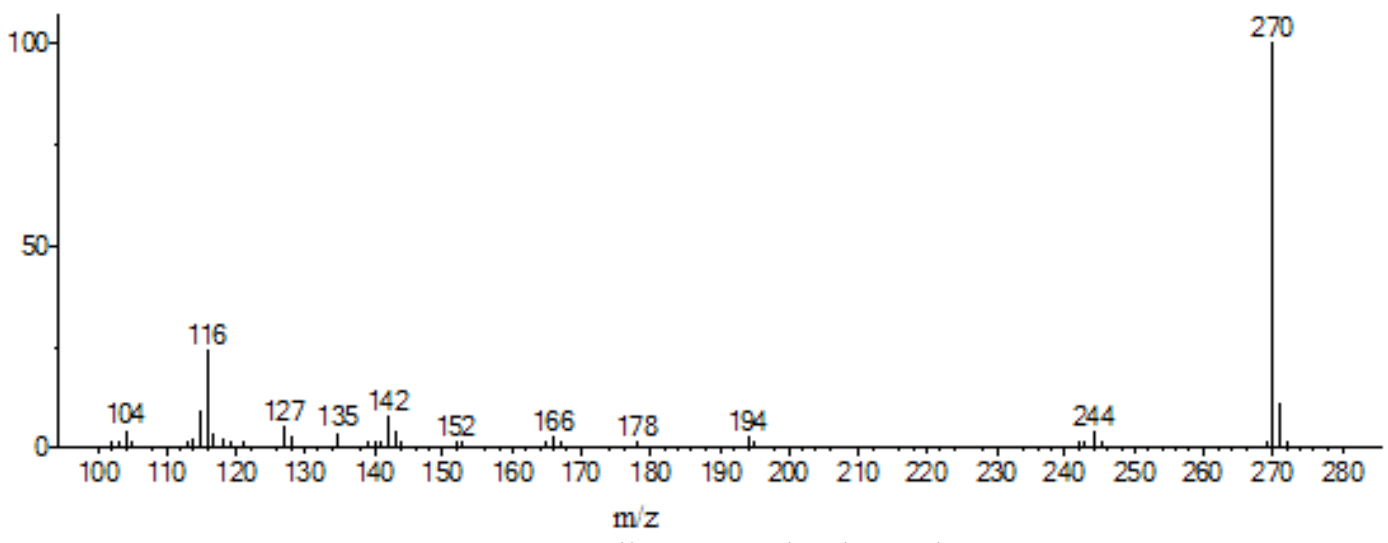

Şekil 4. Ph-IPz ligantının kütle spektrumu

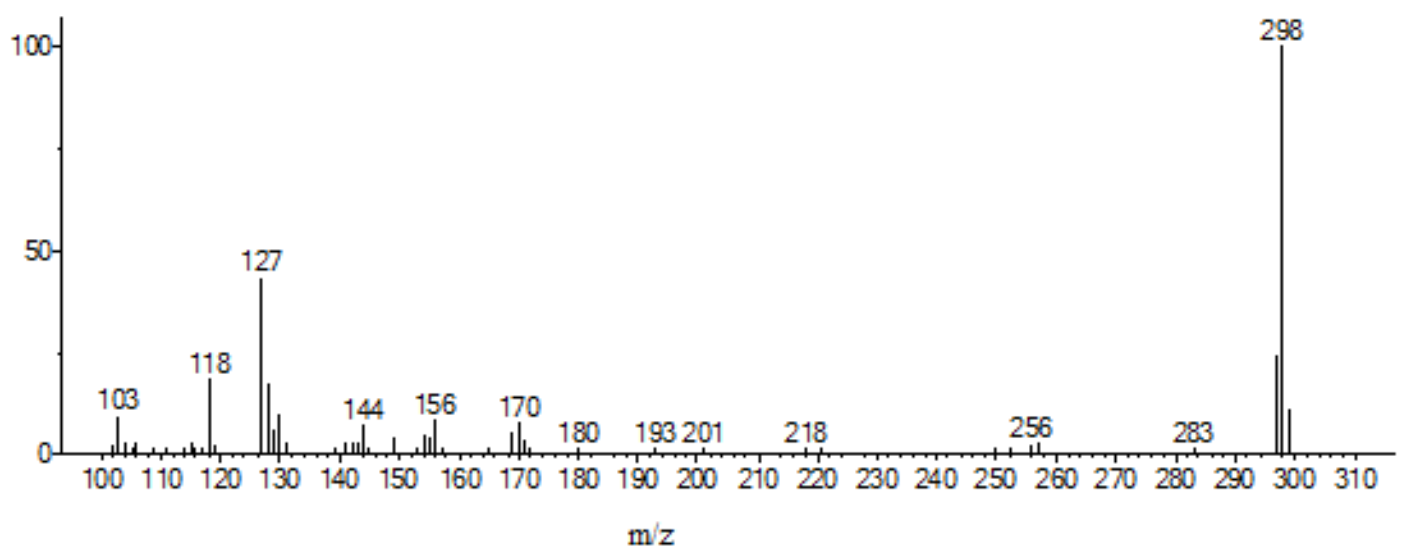

Şekil 5. Ph-IDMPz ligantının kütle spektrumu

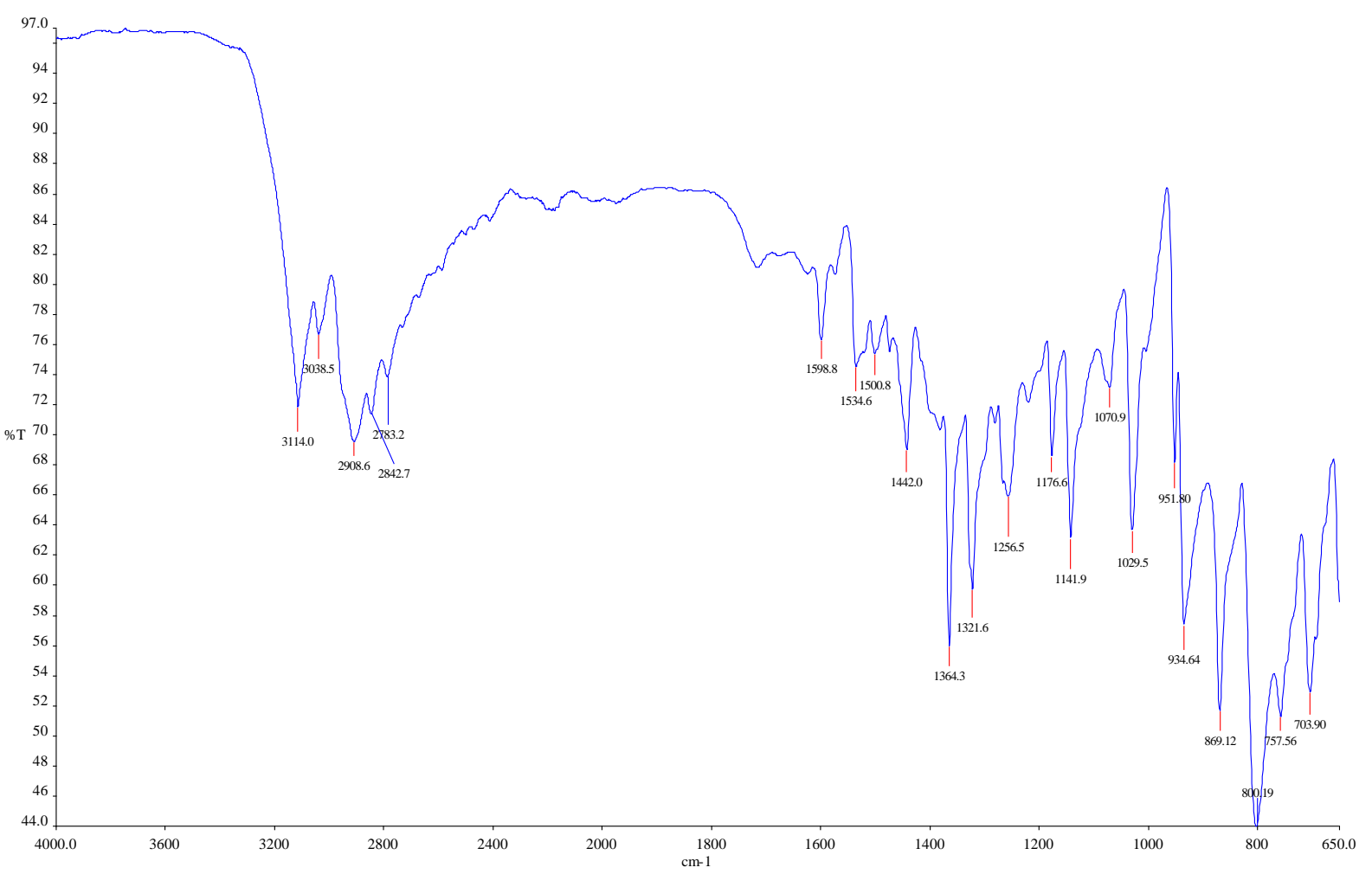

Şekil 6. Ph-IPz ligantının FT-IR spektrumu 


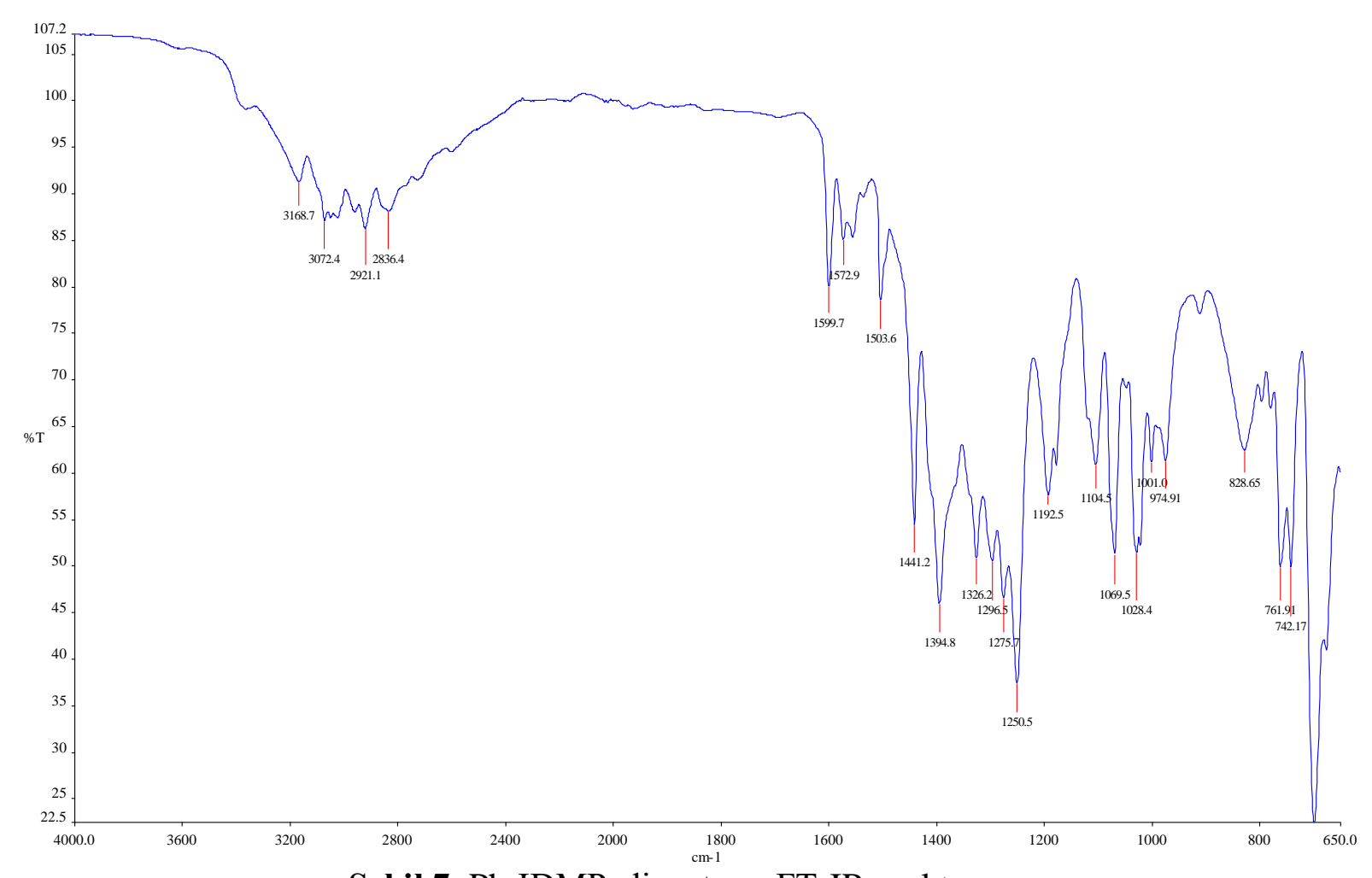

Şekil 7. Ph-IDMPz ligantının FT-IR spektrumu.

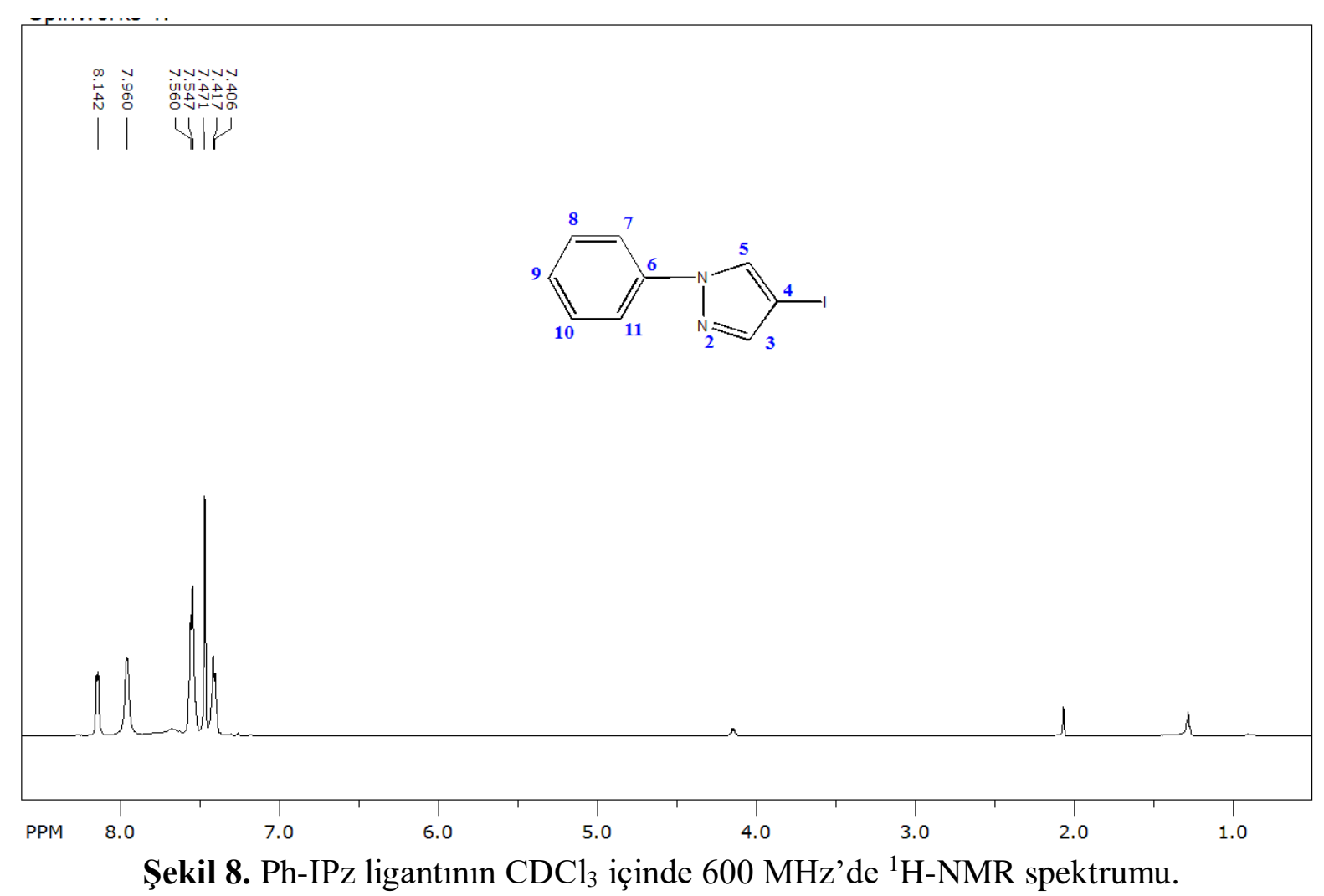




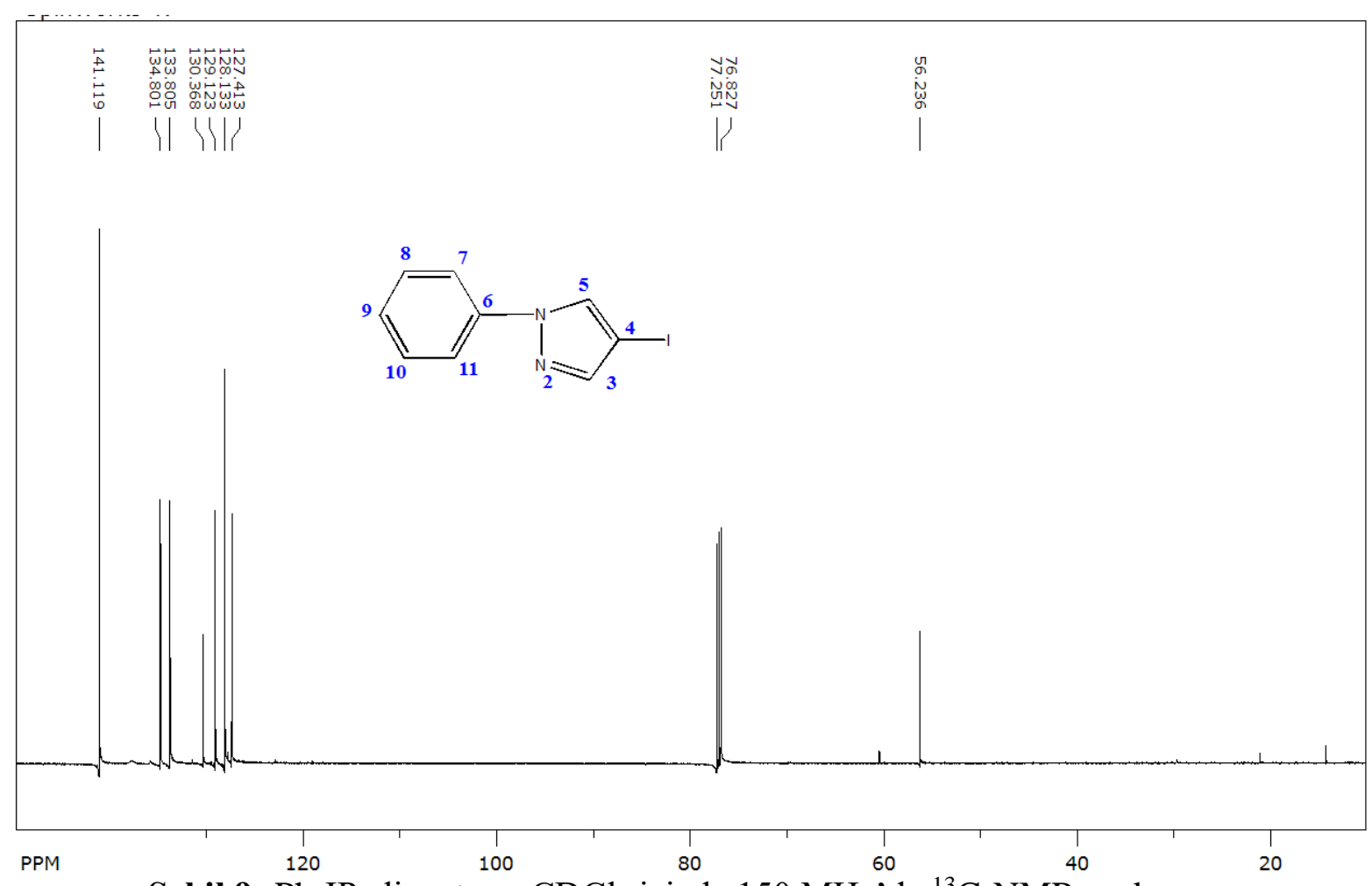

Şekil 9: Ph-IPz ligantının $\mathrm{CDCl}_{3}$ içinde $150 \mathrm{MHz}$ 'de ${ }^{13} \mathrm{C}-\mathrm{NMR}$ spektrumu

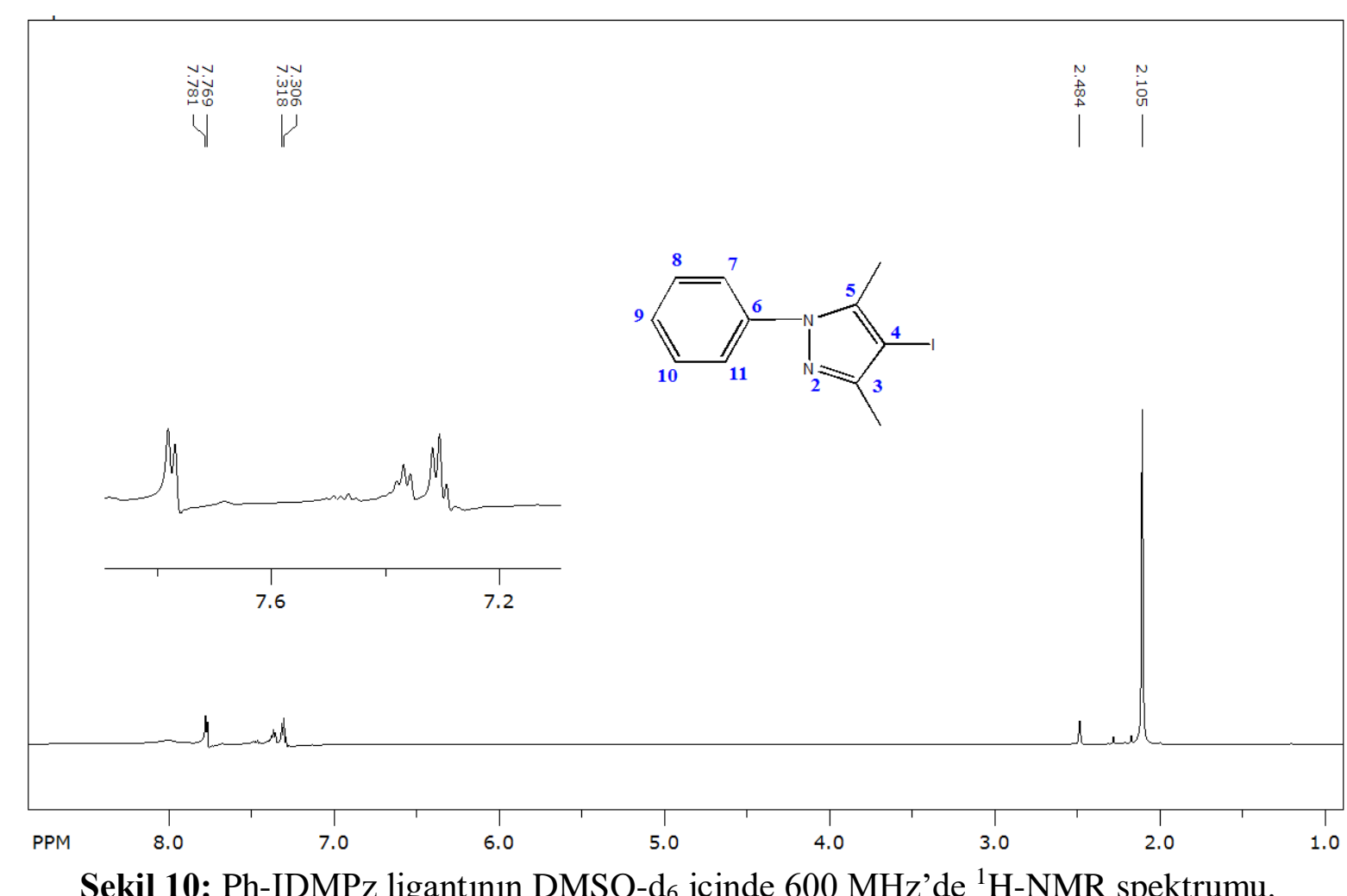

Şekil 10: Ph-IDMPz ligantının DMSO-d $\mathrm{d}_{6}$ içinde $600 \mathrm{MHz}$ 'de ${ }^{1} \mathrm{H}-\mathrm{NMR}$ spektrumu. 


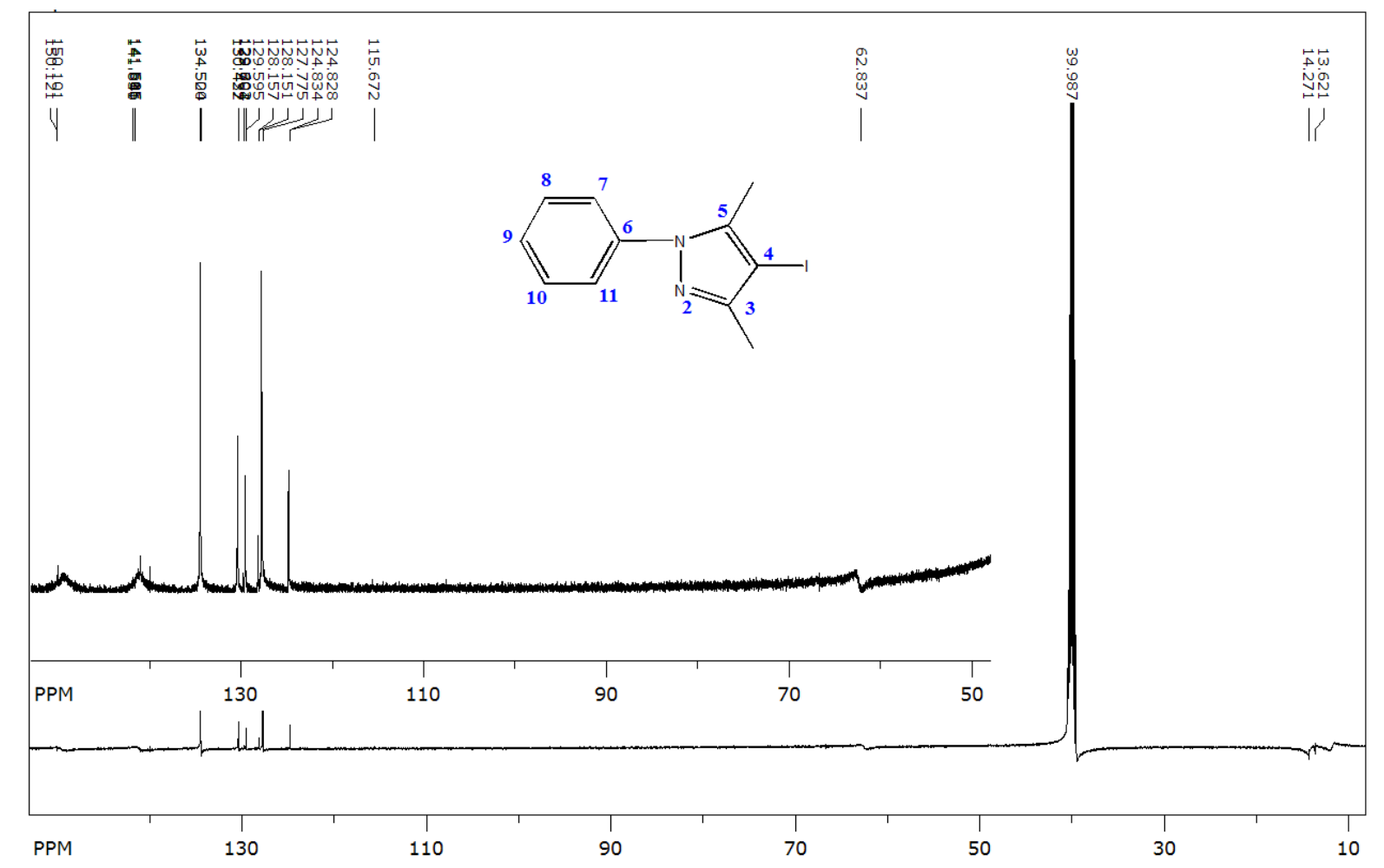

Şekil 11. Ph-IDMPz ligantının DMSO-d $\mathrm{d}_{6}$ içinde $150 \mathrm{MHz} \mathrm{de}^{13} \mathrm{C}-\mathrm{NMR}$ spektrumu.

\section{SONUÇLAR}

Sonuç olarak, $\mathrm{Cu}_{2} \mathrm{O}$ katalizörü ile 4-iyodopirazollerin $\mathrm{N}$-arilasyonunu literatürdeki yöntem uyarlanarak daha kolay, etkili ve ekonomik bir yöntemle elde edildi. Bu yöntemle sert koşullar altında sentezlenen klasik Ullmann reaksiyonunun daha 1lıman koşullar altında ligant veya baz kullanmadan, atmosforik ortamda, oda sıcaklığında ve metanol içerisinde sentezi kolaylıkla gerçekleştirildi. İlk defa Ph-IPz ve Ph-IDMPz bileşiklerinin bu yeni yöntemle elde edilmesi sağlanmış olundu. Başlangıç maddesi I-DMPz’ün yapısında bulunan metil gruplarının elektron verici olması ve metil gruplarının pirazol halkasında simetrik etkiye sahip olmasından dolayı Ph-IDMPz'ün Ph-IPz'e göre veriminin daha fazla ve reaksiyon süresinin daha kısa olduğu görülmüştür. Bileşiklerin yapıları NMR, FT-IR ve GC-MS gibi spektroskopik yöntemlerle aydınlatılmıştır. FT-IR spektrumları başlangıç maddeleri ile karşılaştırıldığında aromatik halkaya özgü karakteristik $\mathrm{C}=\mathrm{N}$ ve $\mathrm{C}=\mathrm{C}$ halka gerilmelerinde belirgin kaymalar olmuştur, buda $\mathrm{N}$-sübstitüe pirazollerin oluşumunun bir göstergesidir. Bileşiklerin NMR spektrumunda hem pirazole hem de fenil grubuna ait karakteristik piklerin gözlenmesi önerilen yapıları desteklemektedir. ${ }^{1} \mathrm{H}-\mathrm{NMR}$ spektrumunda başlangıç maddeleri I-Pz ve I-DMPz ligantlarının $\mathrm{N}-\mathrm{H}$ protonu 13-11 ppm civarında görülmektedir [17, 20-21]. Elde edilen Ph-IPz ve Ph-IDMPz bileşiklerinin ${ }^{1} \mathrm{H}-\mathrm{NMR}$ spektrumlarında N-H proton piklerinin görülmemesi fenil grubunun pirazole NH'dan bağlandığının bir göstergesidir. ${ }^{13} \mathrm{C}-\mathrm{NMR}$ spektrumlarına göre $\mathrm{C}_{4}$ karbonu 4- 
sübstitüe pirazoller için belirleyici olmaktadır. Serbest pirazolde 106.0-105.5 ppm'de görülen $\mathrm{C}_{4}$ karbonu Ph-IPz ve Ph-IDMPz'de sirasiyla 62.8 ve 56.2 ppm'e kadar kayması bu yapıları doğrulamaktadır [2]. Bunlara ilave olarak ligantların erime noktalarının başlangıç maddelerinden farklı olması ve kütle spektrumlarının da beklenen değerlerle aynı olması yapıları ve bu yapıların saflıklarını kesinleştirmiştir.

Bundan sonraki çalışmalarda N- donör ihtiva eden farklı pirazol türevleri ile aril boronik asitlerin reaksiyonu sonucu pek çok bileşik sentezlenebilir. Böylece yeni yöntem sayesinde pirazollerin $\mathrm{N}$-arillasyonu ile geniş etki alanına sahip yeni pirazol türevlerinin sentezlenmesi sağlanabilir.

\section{KAYNAKLAR}

[1] Eicher, T.; Hauptmann, S.; Speicher, The chemistry of heterocycles: structure, reactions, synthesis and applications-3rd edition (Wiley-VCH Verlag GmbH \& Co. KGaA, Weinheim, Germany, 2003)

[2] Wang X.Y., Liu S.Q., Zhang C.Y., Song G., Bai F.Y., Xing Y.H. ve Shi Z., Synthesis, structural, and biological evaluation of the arene-linked pyrazolyl methane ligands and their d9/d10 metal complexes. Polyhedron, 47 (2012) 151-164.

[3] Potapov, A.S., Khlebnikov, A.I., Synthesis of mixed-ligand copper(II) complexes containing bis(pyrazole-1-yl) methane ligands. Polyhedron, 25 (2006) 2683-2690.

[4] Fustero S., Sanchez-Rosello M., Barrio P. ve Simon-Fuentes A., From 2000 to Mid-2010: A fruitful decade for the synthesis of pyrazoles. American Chemical Society, 111 (2011) 6984-7034.

[5] Bouabdallah I., Touzani R., Zidane I., Ramdani A., ve Radi S., Synthesis of some 1-aryl3,5-disubstituted-pyrazoles by $\mathrm{N}$-arylation of 3,5-disubstituted-pyrazoles with 4-fluoro and 2-fluoronitrobenzene under microwave irradiation and classical heating. Arkivoc, 12 (2006) 138-144.

[6] Xu Z.L., Li H.X., Ren Z-G. , Du W.Y., Xu W.C. ve Lang J.P., Cu(OAc) 2.H $_{2} \mathrm{O}$-catalyzed $N$-arylation of nitrogen-containing heterocycles. Tetrahedron, 67 (2011) 5282-5288.

[7] Kaplars A., Antilla J.C., Huang X. ve Buchwald S.L.J., A general and efficient copper catalyst for the amidation of aryl halides and the $N$-arylation of nitrogen heterocycles. Journal of American Chemical Society, 123 (31) (2001) 7727-7729.

[8] Taillefer M., Cristau H.J., Cellier P.P. ve Spindler J.F., Patents FR 2833947-WO0353225 (Pr. Nb. Fr 2001 16547) (2001).

[9] Evano G., Blanchard N. ve Toumi M., Copper-mediated coupling reactions and their applications in natural products and designed biomolecules synthesis. Chemical Reviews, 108 (8) (2008) 3054-3131.

[10]Ley S.V. ve Thomas A.W., Modern synthetic methods for copper-mediated $C($ aryl)[bond]O, C(aryl)[bond]N, and C(aryl)[bond]S bond formation. Angewandte Chemie International Edition, 42 (44) (2003) 5400-5449. 
[11]Kim M. M., Ruck R.T., Zhao D. ve Huffman M.A., Green iodination of pyrazoles with iodine/hydrogen peroxide in water. Tetrahedron Letters, 49 (2008) 4026-4028.

[12] Kantam M.L., Venkanna G.T., Sridhar C., Sreedhar B. ve Choudary B.M., An efficient base-free $n$-arylation of imidazoles and amines with arylboronic acids using copperexchanged fluorapatite. Jornal of Organic Chemistry, 71 (2006) 9522-9524.

[13] Joubert N., Baslé E., Vaultier M. ve Pucheault M., Mild, base-free copper-catalyzed Narylations of heterocycles using potassium aryltrifluoroborates in water under air. Tetrahedron Letters, 51 (2010) 2994-2997.

[14] Rao H., Fu H., Jiang Y.ve Zhao Y., Easy copper-catalyzed synthesis of primary aromatic amines by coupling aromatic boronic acids with aqueous ammonia at room temperature. Angewandte Chemie International Edition, 48 (2009) 1114-1116.

[15] Roy S., Roy S. ve Gribble G.W., Metalation of pyrazoles and indazoles, Topics in Heterocycle Chemistry, 29 (2012) 155-260.

[16]Coates J., Interpretation of Infrared Spectra, A Practical Approach, 10815-10837, R.A. Meyers (Ed.). Encyclopedia of Analytical Chemistry (John Wiley \& Sons Ltd., Coates Consulting, Newtown, USA, 2000)

[17] Skoog D.A., Holler F.J. ve Nieman T.A., Principles of Instrumental Analysis, Fifth Edition (Harcourt Brace \&Company, Florida, USA, 1998)

[18] Stuart B.H., Infrared Spectroscopy: Fundamentals and Applications (John Wiley \& Sons Ltd., İngiltere, 2004).

[19] Sharma S., Barooah N., ve Baruah J.B., Tris (3,5-dimethylpyrazole) copper(II) nitrate: as an oxidation catalyst. Journal of Molecular Catalysis A: Chemical, 229 (2005) 171-176.

[20] Ovejero P., Mayoral M. J., Cano M., Campo J.A., Heras J.V., Pinilla E., Torres M. R., The 3,5-dimethyl-4-nitropyrazole ligand in the construction of supramolecular networks of silver(I) complexes, Journal of Organometallic Chemistry, 692 (2007) 4093-4105.

[21]Mezei G., Raptis R.G., Effect of pyrazole-substitution on the structure and nuclearity of Cu(II)-pyrazolato complexes, Inorganica Chimica Acta, 357 (2004) 3279-3288. 